\title{
Localization of Near-Field Sources Based on Sparse Signal Reconstruction with Regularization Parameter Selection
}

\author{
Shuang Li, ${ }^{1}$ Wei Liu, ${ }^{1}$ Daqing Zheng, ${ }^{1}$ Shunren $\mathrm{Hu},{ }^{1}$ and Wei $\mathrm{He}^{2}$ \\ ${ }^{1}$ School of Electrical and Electronic Engineering, Chongqing University of Technology, Chongqing 400054, China \\ ${ }^{2}$ Key Laboratory of Wireless Sensor Networks and Communication, Shanghai Institute of Microsystem and Information Technology, \\ Shanghai 200050, China \\ Correspondence should be addressed to Wei He; wei.he@mail.sim.ac.cn
}

Received 9 November 2016; Accepted 9 April 2017; Published 11 May 2017

Academic Editor: María Elena de Cos Gómez

Copyright (C) 2017 Shuang Li et al. This is an open access article distributed under the Creative Commons Attribution License, which permits unrestricted use, distribution, and reproduction in any medium, provided the original work is properly cited.

\begin{abstract}
Source localization using sensor array in the near-field is a two-dimensional nonlinear parameter estimation problem which requires jointly estimating the two parameters: direction-of-arrival and range. In this paper, a new source localization method based on sparse signal reconstruction is proposed in the near-field. We first utilize $\ell_{1}$-regularized weighted least-squares to find the bearings of sources. Here, the weight is designed by making use of the probability distribution of spatial correlations among symmetric sensors of the array. Meanwhile, a theoretical guidance for choosing a proper regularization parameter is also presented. Then one well-known $\ell_{1}$-norm optimization solver is employed to estimate the ranges. The proposed method has a lower variance and higher resolution compared with other methods. Simulation results are given to demonstrate the superior performance of the proposed method.
\end{abstract}

\section{Introduction}

Source localization using sensor array is one of the most important topics in array signal processing society. A great number of source localization methods were proposed in the past few decades. However, most of these methods focused on source localization in far-field case, in which the signal can be regarded as planar wave and only direction-of-arrival (DOA) estimation is required. When the range between the sources and the array is not sufficiently large compared with the aperture of the array (i.e., in the near-field case), the wavefront of the signal at the array is characterized by both azimuth and range. Thus, the performance of the DOA estimation methods for far-field case degrades significantly in the near-field.

In recent years, a majority of methods have been proposed to deal with the source localization problem in the nearfiled, such as maximum likelihood methods [1], the twodimensional MUSIC methods [2], high-order-cumulantsbased methods [3-7], and the linear prediction methods [8, 9]. However, most of these methods either require additional parameters pairing $[8,9]$ or involve large computational cost due to multidimensional search $[1,2]$ or the computation of cumulants [3-7]. Furthermore, in order to take advantages of the symmetric property of the array, some other methods suffer from heavy aperture loss (i.e., at most $M$ sources can be detected when the number of sensors is $2 M-1[10,11])$. Recently, Malioutov et al. [12] proposed a DOA estimation method in far-field named L1-SVD, showing some advantages including high resolution and improved robustness to noise, to a limited number of snapshots and to correlation of sources. After that, several methods based on sparse signal reconstruction (SSR) were proposed to locate the near-field sources. Wang et al. [13] proposed a mixed source localization method based on sparse representation of cumulants, achieving a higher estimation accuracy. However, the method suffers from a heavy computational load for computation of cumulants. By representing the source range and DOA information as sensor-dependent phase progression, a Bayesiancompressed-sensing-based source localization method was proposed for uniform and sparse linear arrays [14]. However, it also suffers from large computational cost because of iterations. By jointly using MUSIC and sparse signal reconstruction, Tian and Sun [15] also proposed a source localization 
method for mixed sources. By making use of the spatial correlations of symmetric sensors output, an SSR-based source localization method was proposed by Hu et al. [16, 17], showing superior performance. However, by employing $l_{1}$ regularized least-squares optimization to find the sparse solution, the regularization parameter was selected manually by cross validation, which causes the method to be unable to use in practice. Moreover, it is prone to be selected improperly.

In this paper, a novel SSR-based source localization method is proposed in the near-field. Firstly, just like the method in [16], by exploiting the spatial correlations of symmetric sensors output, the azimuth and range are decoupled so that a two-dimensional parameter estimation problem in the near-field is converted into a DOA estimation one in the far-field. Secondly, the theory of $l_{1}$-regularized weighted least-squares optimization is employed on the virtual farfield array to acquire DOA estimation. Meanwhile, similar to [18], an approach is presented to choose regularization parameter. At last, L1-SVD is utilized to estimate the ranges of the sources.

The paper is organized as follows. Section 2 describes the data model of source localization. An existing SSR-based method for source localization in the near-field is reviewed in Section 3. The proposed method for DOA and range estimation is presented in Sections 4 and 5, respectively. Simulation results are shown in Section 6. Section 7 concludes this paper.

\section{Data Model}

Consider this case in which $K$ near-field narrowband sources impinge onto a uniform linear array with $M=2 q+1$ elements, as depicted in Figure 1. The received signal of $i$ th sensor can be expressed as

$$
\begin{aligned}
x_{i}(t)=\sum_{k=1}^{K} s_{k}(t) e^{j(2 \pi / \lambda)\left(r_{i, k}-r_{k}\right)}+v_{i}(t), \\
\quad i=-q,-q+1, \ldots, q, t=1,2, \ldots, T,
\end{aligned}
$$

where $s_{k}(t)$ represents the $k$ th source signal, $v_{i}(t)$ denotes the additive noise received by the $i$ th sensor, $r_{i, k}$ stands for the range between the $k$ th source and the $i$ th sensor, $r_{k}$ refers to the range between the $k$ th source and the reference sensor of the array, $\lambda$ is the wavelength of the narrowband signals, and $T$ denotes the number of snapshots. It can be easily derived from Figure 1 that

$$
r_{i, k}=\sqrt{r_{k}^{2}+i^{2} d^{2}-2 i d \sin \theta_{k}}
$$

where $\theta_{k}$ denotes the DOA of the $k$ th source.

Let $\mathbf{x}(t)=\left[x_{-q}(t), x_{-q+1}(t), \ldots, x_{q}(t)\right]^{T}, \mathbf{s}(t)=\left[s_{1}(t)\right.$, $\left.s_{2}(t), \ldots, s_{K}(t)\right]^{T}$, and $\mathbf{v}(t)=\left[v_{-q}(t), v_{-q+1}(t), \ldots, v_{q}(t)\right]^{T}$ denote the received signal vector, the source signal vector, and the noise vector, respectively. By stacking all $\left\{x_{i}(t), i=\right.$ $-q,-q+1, \ldots, q\}$ into a vector, we arrive at

$$
\mathbf{x}(t)=\mathbf{B s}(t)+\mathbf{v}(t), \quad t=1,2, \ldots, T,
$$

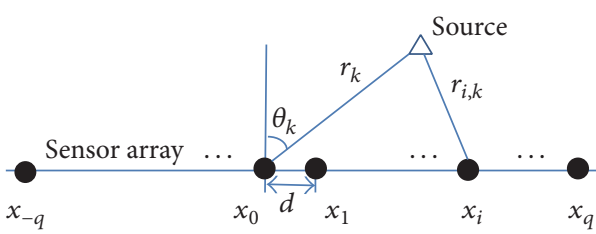

FIgURE 1: A near-field source impinges onto a ULA with $M=2 q+1$ sensors.

where $\mathbf{B}=\left[\mathbf{b}\left(\theta_{1}, r_{1}\right), \mathbf{b}\left(\theta_{2}, r_{2}\right), \ldots, \mathbf{b}\left(\theta_{K}, r_{K}\right)\right]$ denotes the array manifold and $\mathbf{b}\left(\theta_{k}, r_{k}\right)=\left[e^{j(2 \pi / \lambda)\left(r_{-q, k}-r_{k}\right)}, e^{j(2 \pi / \lambda)\left(r_{-q+1, k}-r_{k}\right)}, \ldots\right.$, $\left.e^{j(2 \pi / \lambda)\left(r_{q, k}-r_{k}\right)}\right]$ stands for the steering vector.

By making use of the Fresnel approximation [16], $r_{i, k}-r_{k}$ can be written as

$$
r_{i, k}-r_{k} \approx-i d \sin \theta_{k}+\frac{i^{2} d^{2}}{2 r_{k}} \cos \theta_{k}^{2}
$$

If we define $\omega_{k}=-(2 \pi / \lambda) d \sin \theta_{k}$ and $\varphi_{k}=\left(\pi d^{2} / \lambda r_{k}\right) \cos ^{2} \theta_{k}$, the steering vector can be approximated as

$$
\begin{aligned}
\mathbf{b} & \left(\theta_{k}, r_{k}\right) \approx \mathbf{a}\left(\omega_{k}, \varphi_{k}\right) \\
& =\left[e^{j(-q) \omega_{k}+(-q)^{2} \varphi_{k}}, e^{j(-q+1) \omega_{k}+(-q+1)^{2} \varphi_{k}}, \ldots, e^{j q \omega_{k}+q^{2} \varphi_{k}}\right]^{T}
\end{aligned}
$$

which indicates

$$
\mathbf{B} \approx \mathbf{A},
$$

where $\mathbf{A}=\left[\mathbf{a}\left(\omega_{1}, \varphi_{1}\right), \mathbf{a}\left(\omega_{2}, \varphi_{2}\right), \ldots, \mathbf{a}\left(\omega_{K}, \varphi_{K}\right)\right]$ denotes the approximated array manifold. Substituting (6) into (3), we arrive at

$$
\mathbf{x}(t)=\mathbf{A s}(t)+\mathbf{v}(t), \quad t=1,2, \ldots, T
$$

Given the knowledge of the observed signals $\{\mathbf{x}(t), t=$ $1,2, \ldots, T\}$, the goal is to find the ranges $\mathbf{r}=\left[r_{1}, r_{2}, \ldots, r_{k}\right]^{T}$ and the DOAs $\boldsymbol{\theta}=\left[\theta_{1}, \theta_{2}, \ldots, \theta_{K}\right]^{T}$.

For convenience we make the following assumptions:

(A1) The source signals are uncorrelated to each other and independent of the noise.

(A2) The noises are spatially uncorrelated Gaussian white noise.

(A3) The intersensor spacing of the array $d$ satisfies $d \leq$ $\lambda / 4$.

\section{The DOA Estimation Method in [16]}

Under the above assumptions, the spatial correlation between the $m$ th and the $n$th sensor output can be expressed as

$$
\begin{aligned}
r_{x}(m, n) & =E\left\{x_{m}(t) x_{n}^{*}(t)\right\} \\
& =\sum_{k=1}^{K} \sigma_{s, k}^{2} e^{j(m-n) \omega_{k}+j\left(m^{2}-n^{2}\right) \varphi_{k}}+\delta(m-n) \sigma_{v}^{2},
\end{aligned}
$$


where $\sigma_{s, k}^{2}$ stands for the power of the $k$ th source signal, $\sigma_{v}^{2}$ represents the noise power, and $\delta(\cdot)$ denotes the Dirac function. Note that when $n=-m$, the spatial correlation is uncorrelated with the parameter $\varphi_{k}$. Thus, we have

$$
r_{x}(m,-m)=\sum_{k=1}^{K} \sigma_{s, k}^{2} e^{j(2 m) \omega_{k}}+\delta(2 m) \sigma_{v}^{2}
$$

Stacking all $r_{x}(m,-m)$ from $-q$ to $q$, we obtain

$$
\mathbf{r}_{x}=\tilde{\mathbf{A}}(\omega) \mathbf{r}_{s}+\sigma_{v}^{2} \mathbf{e}
$$

where $\mathbf{r}_{x}=\left[r_{x}(-q, q), r_{x}(-q+1, q-1), \ldots, r_{x}(q,-q)\right]^{T}, \mathbf{r}_{s}=$ $\left[\sigma_{s, 1}^{2}, \sigma_{s, 2}^{2}, \ldots, \sigma_{s, K}^{2}\right]^{T}, \mathbf{e}=\left[\mathbf{0}_{q}^{T}, 1, \mathbf{0}_{q}^{T}\right]^{T}$, and $\widetilde{\mathbf{A}}(\omega)=\left[\widetilde{\mathbf{a}}\left(\omega_{1}\right)\right.$, $\left.\widetilde{\mathbf{a}}\left(\omega_{2}\right), \ldots, \widetilde{\mathbf{a}}\left(\omega_{K}\right)\right], \widetilde{\mathbf{a}}\left(\omega_{k}\right)=\left[e^{-j 2 q \omega_{k}}, e^{-j 2(q-1) \omega_{k}}, \ldots, e^{j 2 q \omega_{k}}\right]^{T}$. Comparing (10) with (7), $\mathbf{r}_{x}$ behaves like the received signal of a far-field array with array manifold $\widetilde{\mathbf{A}}(\omega)$ and source signal $\mathbf{r}_{s}$, corrupted by the noise $\sigma_{v}^{2}$ e. Note that the two-dimensional (DOA and range) estimation problem has been transformed into a one-dimensional (DOA) estimation one now.

Then, the one-dimensional estimation problem can be cast into a sparse signal recovery problem as follows. Define a set $\Theta=\left\{\bar{\theta}_{1}, \bar{\theta}_{2}, \ldots, \bar{\theta}_{N_{\theta}}\right\}$ as the sampling grid corresponding to the DOAs of the potential sources. The number of the potential sources $N_{\theta}$ should be much greater than the number of the real sources $K$ and the number of sensors $M$. The overcomplete basis $\overline{\mathbf{A}}(\omega)$ can be constructed as

$$
\overline{\mathbf{A}}(\omega)=\left[\widetilde{\mathbf{a}}\left(\bar{\omega}_{1}\right), \widetilde{\mathbf{a}}\left(\bar{\omega}_{2}\right), \ldots, \widetilde{\mathbf{a}}\left(\bar{\omega}_{N_{\theta}}\right)\right]
$$

where $\bar{\omega}_{i}=-(2 \pi / \lambda) d \sin \bar{\theta}_{i}$. The sparse signal is represented by a vector $\mathbf{p} \in C^{N_{\theta} \times 1}$, whose $i$ th element should be a nonzero weight $p_{i}=\sigma_{s, k}^{2}$ if the $k$ th source comes from the direction $\bar{\theta}_{i}$ for some $k$ and zero otherwise; that is, the sparse vector $\mathbf{p}$ acts as the spatial spectrum. Thus, the sparse signal recovery model is formulated as

$$
\mathbf{r}_{x}=\overline{\mathbf{A}}(\omega) \mathbf{p}+\sigma_{v}^{2} \mathbf{e}
$$

A usual way to solve the sparse signal $\mathbf{p}$ is using the wellknown $\ell_{1}$-regularized least-squares minimization method, which is given by [16]

$$
\widehat{\mathbf{p}}=\underset{\mathbf{p}}{\arg \min }\left\|\mathbf{r}_{x}-\overline{\mathbf{A}}(\omega) \mathbf{p}-\sigma_{v}^{2} \mathbf{e}\right\|_{2}^{2}+\beta\|\mathbf{p}\|_{1},
$$

where $\beta$ denotes the regularization parameter, which balances the data-fitting error and the sparsity of $\mathbf{p}$. It is important to select the parameter $\beta$ properly since it has a great impact on the spatial spectrum. If the parameter $\beta$ is too small, some of the peaks in the spatial spectrum will disappear. On the contrary, spurious peaks arise when the parameter $\beta$ is too large. In [16], the parameter is selected manually by cross validation, which not only causes inconvenience and improper selection but also results in unavailability in practice.

\section{The Proposed DOA Estimation Method}

Under the assumptions (A1) and (A2) in Section 2, the covariance matrix of the received signals can be expressed as

$$
\mathbf{R}=\mathbf{A} \Sigma_{s} \mathbf{A}^{H}+\sigma_{v}^{2} \mathbf{I}
$$

where $\Sigma_{s}=\operatorname{diag}\left(\mathbf{r}_{s}\right)$. In practice, the real covariance matrix is unavailable; however, it can be consistently estimated by

$$
\widehat{\mathbf{R}}=\frac{1}{T} \sum_{t=1}^{T} \mathbf{x}(t) \mathbf{x}^{H}(t) .
$$

The estimate error is

$$
\Delta \mathbf{R}=\widehat{\mathbf{R}}-\mathbf{R} .
$$

Without consideration of the error caused by Fresnel approximation, the vector form of $\Delta \mathbf{R}$ satisfies [19]

$$
\Delta \mathbf{r}_{v} \triangleq \operatorname{vec}(\Delta \mathbf{R}) \sim \operatorname{AsN}\left(0, \frac{1}{T} \mathbf{R}^{T} \otimes \mathbf{R}\right),
$$

where vec $(\cdot)$ refers to the vectorization operation, $\operatorname{AsN}\left(\mu, \sigma^{2}\right)$ represents asymptotic normal distribution with mean $\mu$ and variance $\sigma^{2}$, and the symbol $\otimes$ denotes Kronecker product.

Define $\mathbf{r}_{v} \triangleq \operatorname{vec}(\mathbf{R})$. It can be verified that the two vectors $\mathbf{r}_{x}$ and $\mathbf{r}_{v}$ satisfy

$$
\mathbf{r}_{x}(M+1-i)=\mathbf{r}_{v}(i(M-1)+1), \quad i=1,2, \ldots, M .
$$

Let $\widehat{\mathbf{r}}_{x}$ denote the estimate of $\mathbf{r}_{x}$ and $\widehat{\mathbf{r}}_{v}=\operatorname{vec}(\widehat{\mathbf{R}})$ be the estimate of $\mathbf{r}_{v}=\operatorname{vec}(\mathbf{R})$; obviously, we have

$$
\widehat{\mathbf{r}}_{x}(M+1-i)=\widehat{\mathbf{r}}_{v}(i(M-1)+1), \quad i=1,2, \ldots, M .
$$

The estimation error of $\mathbf{r}_{x}$ is defined as

$$
\Delta \mathbf{r}_{x}=\widehat{\mathbf{r}}_{x}-\mathbf{r}_{x} .
$$

Then, the $(M+1-i)$ th element of the estimation error $\Delta \mathbf{r}_{x}$ can be written as

$$
\begin{aligned}
& \Delta \mathbf{r}_{x}(M+1-i)=\widehat{\mathbf{r}}_{x}(M+1-i)-\mathbf{r}_{x}(M+1-i) \\
& =\widehat{\mathbf{r}}_{v}(i(M-1)+1) \\
& -\mathbf{r}_{v}(i(M-1)+1) \\
& =\Delta \mathbf{r}_{v}(i(M-1)+1) \text {, } \\
& i=1,2, \ldots, M
\end{aligned}
$$

which implies

$$
\Delta \mathbf{r}_{x}=\mathbf{C} \Delta \mathbf{r}_{v}
$$

where

$$
\begin{aligned}
\mathbf{C} & =\left[\begin{array}{cccc|cccc|c|cccc}
0 & \cdots & 0 & 0 & 0 & \cdots & 0 & 0 & \cdots & 1 & 0 & \cdots & 0 \\
\vdots & \ddots & \vdots & \vdots & \vdots & \ddots & \vdots & \vdots & \cdots & 0 & 0 & \cdots & 0 \\
0 & \cdots & 0 & 0 & 0 & \cdots & 1 & 0 & \cdots & \vdots & \ddots & \vdots & \vdots \\
0 & \cdots & 0 & 1 & 0 & \cdots & 0 & 0 & \cdots & 0 & 0 & \cdots & 0
\end{array}\right] \\
& \in R^{M \times M^{2}} .
\end{aligned}
$$


By taking advantages of the property of normal distribution, it can be derived from (17) and (22) that

$$
\Delta \mathbf{r}_{x} \sim \operatorname{AsN}\left(0, \frac{1}{T} \mathbf{C}\left(\mathbf{R}^{T} \otimes \mathbf{R}\right) \mathbf{C}^{T}\right) .
$$

To fit the data $\widehat{\mathbf{r}}_{x}$ to its data model well while finding the sparsest solution $\mathbf{p}$, it is better to employ the weighted leastsquares method; that is,

$$
\begin{aligned}
\min _{\mathbf{p}} & \left(\widehat{\mathbf{r}}_{x}-\overline{\mathbf{A}}(\omega) \mathbf{p}-\sigma_{v}^{2} \mathbf{e}\right)^{H} \mathbf{W}^{-1}\left(\widehat{\mathbf{r}}_{x}-\overline{\mathbf{A}}(\omega) \mathbf{p}-\sigma_{v}^{2} \mathbf{e}\right) \\
& +\alpha\|\mathbf{p}\|_{1}
\end{aligned}
$$

or

$$
\min _{\mathbf{p}}\left\|\mathbf{W}^{-1 / 2}\left(\widehat{\mathbf{r}}_{x}-\overline{\mathbf{A}}(\omega) \mathbf{p}-\sigma_{v}^{2} \mathbf{e}\right)\right\|_{2}^{2}+\alpha\|\mathbf{p}\|_{1},
$$

where $\mathbf{W}$ is a weighted matrix, $\mathbf{W}^{-1 / 2}$ denotes the Hermitian square root of $\mathbf{W}^{-1}$, and $\alpha$ is regularization parameter. As stated before, it is of significant importance to select the regularization parameter properly. Here, similar to [18], an approach to choose $\alpha$ is given as follows.

In order to fit the data $\widehat{\mathbf{r}}_{x}$ to its data model well, $\mathbf{W}$ is set as asymptotic covariance matrix of $\Delta \mathbf{r}_{x}$; that is, $\mathbf{W}=\mathbf{C}\left(\mathbf{R}^{T} \otimes\right.$ R) $\mathbf{C}^{T} / T$. From (24), it can be derived that

$$
\mathbf{W}^{-1 / 2}\left(\widehat{\mathbf{r}}_{x}-\overline{\mathbf{A}}(\omega) \mathbf{p}-\sigma_{v}^{2} \mathbf{e}\right) \sim \operatorname{AsN}(0,1) .
$$

Hence, we obtain

$$
\left\|\mathbf{W}^{-1 / 2}\left(\widehat{\mathbf{r}}_{x}-\overline{\mathbf{A}}(\omega) \mathbf{p}-\sigma_{v}^{2} \mathbf{e}\right)\right\|_{2}^{2} \sim \operatorname{As} \chi^{2}(M),
$$

where $A s \chi^{2}(M)$ represents the asymptotic chi-square distribution with $M$ degrees of freedom. To solve the problem in (26), we introduce another parameter $\gamma$ and should choose it high enough so that the probability of $\| \mathbf{W}^{-1 / 2}\left(\widehat{\mathbf{r}}_{x}-\overline{\mathbf{A}}(\omega) \mathbf{p}-\right.$ $\left.\sigma_{v}^{2} \mathbf{e}\right) \|_{2}^{2} \geq \gamma$ is small, which implies that

$$
\left\|\mathbf{W}^{-1 / 2}\left(\widehat{\mathbf{r}}_{x}-\overline{\mathbf{A}}(\omega) \mathbf{p}-\sigma_{v}^{2} \mathbf{e}\right)\right\|_{2}^{2} \leq \gamma
$$

holds true with a high probability $p_{h}$. We can find a confidence interval for $\left\|\mathbf{W}^{-1 / 2} \Delta \mathbf{r}_{x}\right\|_{2}^{2}$ and use its lower bound as a choice of $\gamma$. Generally, it is enough to choose $p_{h}=0.999$ to determine the parameter $\gamma$.

Now, the problem in (26) can be converted into

$$
\min _{\mathbf{p}}\|\mathbf{p}\|_{1}
$$

subject to $\left\|\mathbf{W}^{-1 / 2}\left(\widehat{\mathbf{r}}_{x}-\overline{\mathbf{A}}(\omega) \mathbf{p}-\sigma_{v}^{2} \mathbf{e}\right)\right\|_{2}^{2} \leq \gamma$,

where $\mathbf{W}^{-1 / 2}=\sqrt{T}\left[\mathbf{C}\left(\mathbf{R}^{T} \otimes \mathbf{R}\right) \mathbf{C}^{T}\right]^{-1 / 2}$ and $\|\cdot\|_{F}$ denotes the Frobenius norm. Equation (30) can be solved by a MATLAB toolbox named CVX [20].

\section{Range Estimation}

In this section, the approach of L1-SVD [12] is exploited to estimate the range of the sources. Let $\widehat{\boldsymbol{\theta}}$ denote the estimated
DOAs from previous section. We define the potential source $\operatorname{grid}$ as $(\widehat{\boldsymbol{\theta}}, \overline{\mathbf{r}})=\left\{\left(\hat{\theta}_{1}, \bar{r}_{1}\right),\left(\widehat{\theta}_{1}, \bar{r}_{2}\right), \ldots,\left(\widehat{\theta}_{1}, r_{N_{r}}\right),\left(\widehat{\theta}_{2}, r_{1}\right), \ldots\right.$, $\left.\left(\widehat{\theta}_{K}, r_{N_{r}}\right)\right\}$ to construct an overcomplete basis $\overline{\mathbf{B}}(\widehat{\boldsymbol{\theta}}, \overline{\mathbf{r}})=$ $\left[\mathbf{b}\left(\widehat{\theta}_{1}, \bar{r}_{1}\right), \mathbf{b}\left(\widehat{\theta}_{1}, \bar{r}_{2}\right), \ldots, \mathbf{b}\left(\widehat{\theta}_{1}, r_{N_{r}}\right), \mathbf{b}\left(\widehat{\theta}_{2}, r_{1}\right), \ldots, \mathbf{b}\left(\widehat{\theta}_{K}, r_{N_{r}}\right)\right] \epsilon$ $C^{M \times K N_{r}}$, where $N_{r}$ is the number of the potential ranges. The source locations are assumed to happen to be located at the grid. Then, the observed signals can be rewritten into a matrix form as

$$
\mathbf{X}=\overline{\mathbf{B}}(\widehat{\boldsymbol{\theta}}, \overline{\mathbf{r}}) \mathbf{S}+\mathbf{V}
$$

where $\mathbf{X}=[\mathbf{x}(1), \mathbf{x}(2), \ldots, \mathbf{x}(T)], \mathbf{V}=[\mathbf{v}(1), \mathbf{v}(2), \ldots, \mathbf{v}(T)]$, and $\mathbf{S}$ is a row sparse matrix, the $\left[(i-1) N_{r}+j\right]$ th row of which is nonzero and equal to a vector $\left[s_{k}(1), s_{k}(2), \ldots, s_{k}(T)\right]$ if a source $k$ comes from $\left(\widehat{\theta}_{i}, \bar{r}_{j}\right)$ for some $k$ and a zero vector otherwise. In order to reduce the computational cost and the influence of noise, we use the singular value decomposition (SVD) of the received signal matrix $\mathbf{X}$. Take the SVD

$$
\mathbf{X}=\mathbf{U L F}^{H}
$$

to decompose the data matrix into signal and noise subspaces and keep a reduced $M \times K$ dimensional matrix $\mathbf{X}_{S V}$, which represents the signal subspace $\mathbf{X}_{S V}=\mathbf{U L D}_{K}=\mathbf{X F D}_{K}$, where $\mathbf{D}_{K}=\left[\mathbf{I}_{K} \mathbf{0}^{T}\right]$. Here, $\mathbf{I}_{K}$ refers to a $K \times K$ identity matrix and $\mathbf{0}$ is a $K \times(T-K)$ matrix of zeros. Furthermore, let $\mathbf{S}_{S V}=\mathbf{S F D}_{K}$ and $\mathbf{V}=\mathbf{V F D}_{K}$; then we can obtain

$$
\mathbf{X}_{S V}=\overline{\mathbf{B}}(\widehat{\boldsymbol{\theta}}, \overline{\mathbf{r}}) \mathbf{S}_{S V}+\mathbf{V}_{S V}
$$

which can be written into a vector form as

$$
\mathbf{x}_{S V}(k)=\overline{\mathbf{B}}(\widehat{\boldsymbol{\theta}}, \overline{\mathbf{r}}) \mathbf{s}_{S V}(k)+\mathbf{v}_{S V}(k), \quad k=1,2, \ldots, K .
$$

Apparently, the two matrices $\mathbf{X}$ and $\mathbf{X}_{S V}$ share the common row sparsity. However, the difference between the two matrices is that the column of the matrix $\mathbf{X}$ is indexing by time samples while that of matrix $\mathbf{X}_{S V}$ is indexing by singular vector number. To solve the sparse signal recovery problem, the $\ell_{2}$ norm of all singular vector index of a particular spatial index of $\mathbf{s}_{S V}(k)$ is calculated first; that is, $s_{i}^{\left(\ell_{2}\right)}=$ $\sqrt{\sum_{k=1}^{K}\left(s_{i}^{S V}(k)\right)^{2}}$; then we impose $\ell_{1}$ norm penalty into all $s_{i}^{\left(\ell_{2}\right)}$ for $i=1,2, \ldots, K N_{r}$. As a result, we can estimate the sparse matrix by minimizing the cost function

$$
\left\|\mathbf{X}_{S V}-\overline{\mathbf{B}}(\widehat{\boldsymbol{\theta}}, \overline{\mathbf{r}}) \mathbf{S}_{S V}\right\|_{F}^{2}+\beta_{r}\left\|\mathbf{s}_{S V}^{\left(\ell_{2}\right)}\right\|_{1}
$$

where $\mathbf{s}_{S V}^{\left(\ell_{2}\right)}=\left[s_{1}^{\left(\ell_{2}\right)}, s_{2}^{\left(\ell_{2}\right)}, \ldots, s_{K N_{r}}^{\left(\ell_{2}\right)}\right]^{T} \in \mathbb{R}^{K N_{r} \times 1}$. The ranges can be obtained by finding the largest $K$ peaks of $\mathbf{s}_{S V}^{\left(\ell_{2}\right)}$ once the matrix $S_{S V}$ is acquired.

\section{Simulation Results}

In this section, some numerical experiments are given to show the effectiveness and efficiency of the proposed method. We make a comparison in terms of RMSE and resolution ability between the proposed method and the method in [16], both of which are based on the theory of sparse signal recovery. In the following simulations, the sources and noises are modeled as white Gaussian signals temporally and spatially, and 200 Monte Carlo trials are performed to calculate the average result for each experiment. 


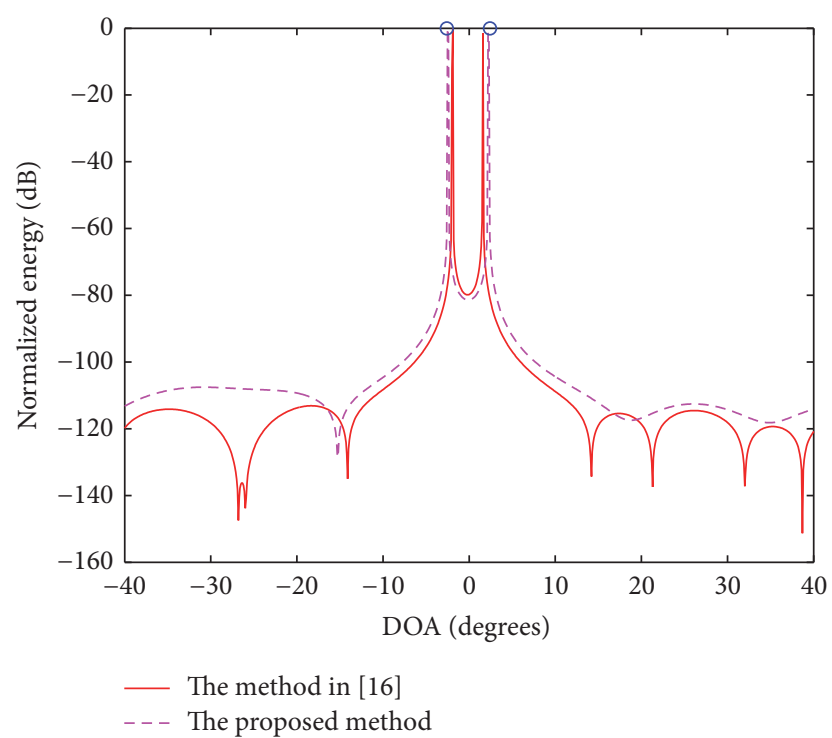

Figure 2: Angular spatial spectra for the two methods.

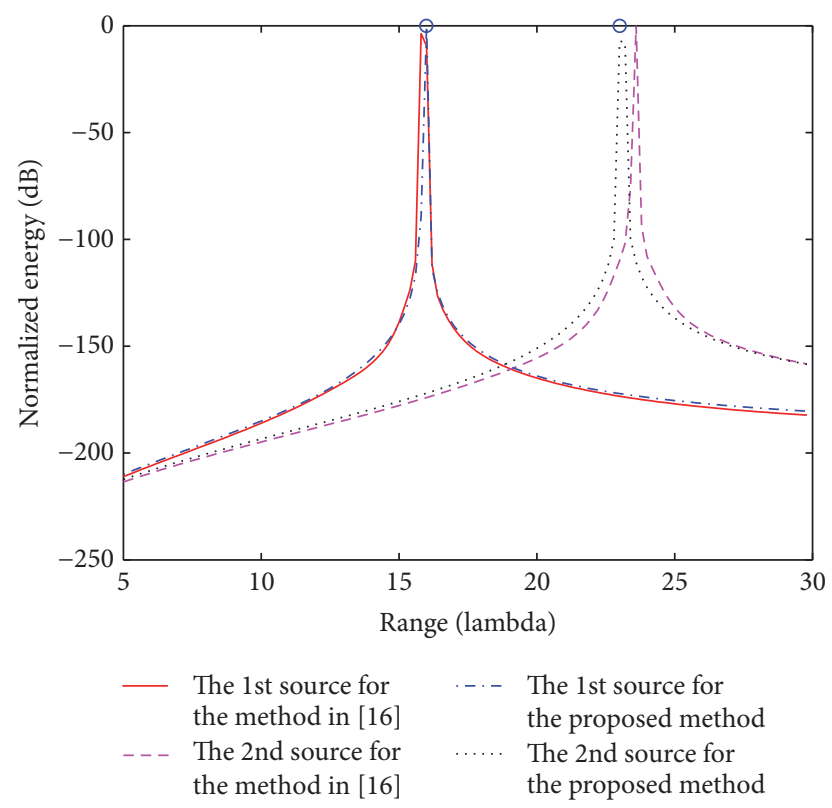

FIGURE 3: Range spatial spectra for the two methods.

6.1. Spatial Spectra. Firstly, we present an experiment to compare the proposed method with the method in [16] in terms of spatial spectra. Consider that two closely spaced signals located at $\left\{16 \lambda,-2.6^{\circ}\right\}$ and $\left\{23 \lambda, 2.4^{\circ}\right\}$ impinge on a ULA with 15 sensors. The intersensor spacing of the ULA is assumed to be $\lambda / 4$. The angular and range spatial spectra are depicted in Figures 2 and 3, respectively.

According to Figures 1 and 2, it can be noted that the proposed method shows the same sharp peaks as the method in [16]. However the proposed method achieves lower errors for range and DOA estimation compared to the method in [16].

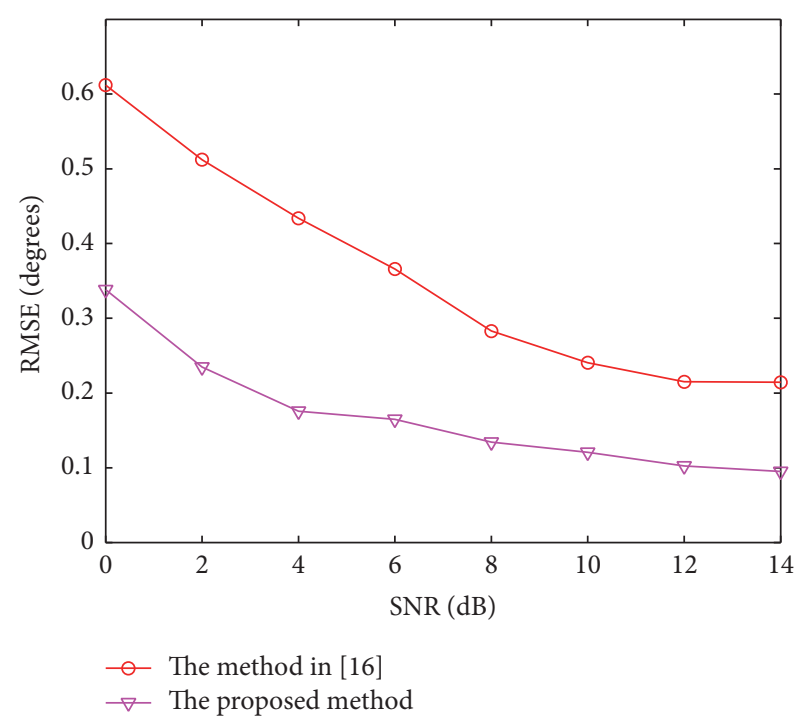

FIgURE 4: RMSE of DOA estimation versus SNR.

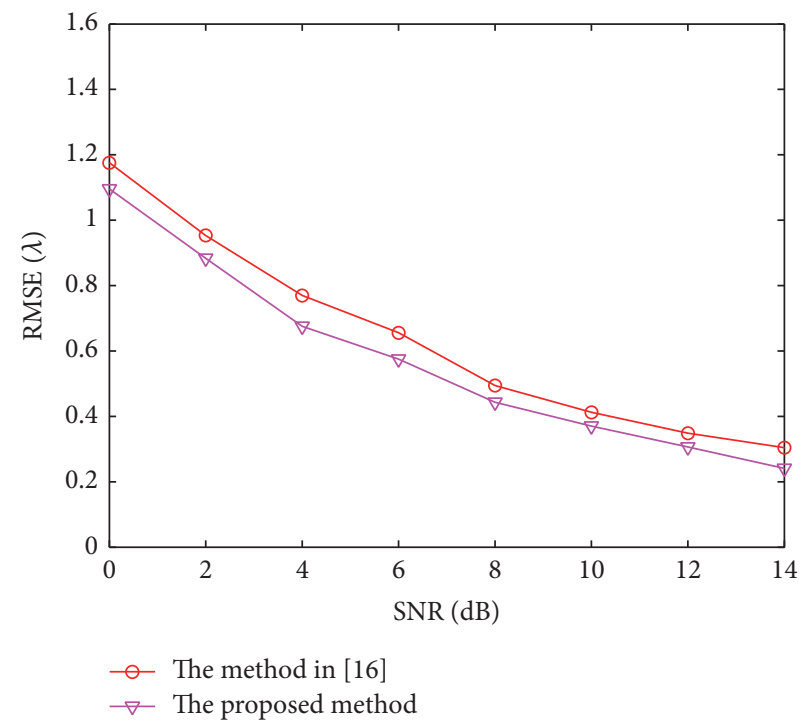

FIGURE 5: RMSE of range estimation versus SNR.

6.2. RMSE versus SNR. Subsequently, we investigate RMSE of DOA and range estimation versus SNR. To make a fair comparison, the two near-field sources are moved to $\left\{17 \lambda,-18.3^{\circ}\right\}$ and $\left\{11 \lambda, 5.6^{\circ}\right\}$. When SNR varies from $0 \mathrm{~dB}$ to $14 \mathrm{~dB}$ with a step $2 \mathrm{~dB}$, by averaging 200 snapshots, we depict the RMSE of DOA and range estimation in Figures 4 and 5, respectively. It can be clearly seen that the proposed method achieves a lower estimate error compared to the method in [16] for both DOA and range estimation.

6.3. RMSE versus the Number of Snapshots. In the second experiment, we evaluate RMSE of DOA and range estimation as a function of the number of snapshots. The parameters are kept the same as before except SNR $=10 \mathrm{~dB}$; the RMSE of DOA and range estimation with respect to the number of snapshots are illustrated in Figures 6 and 7. According to the 


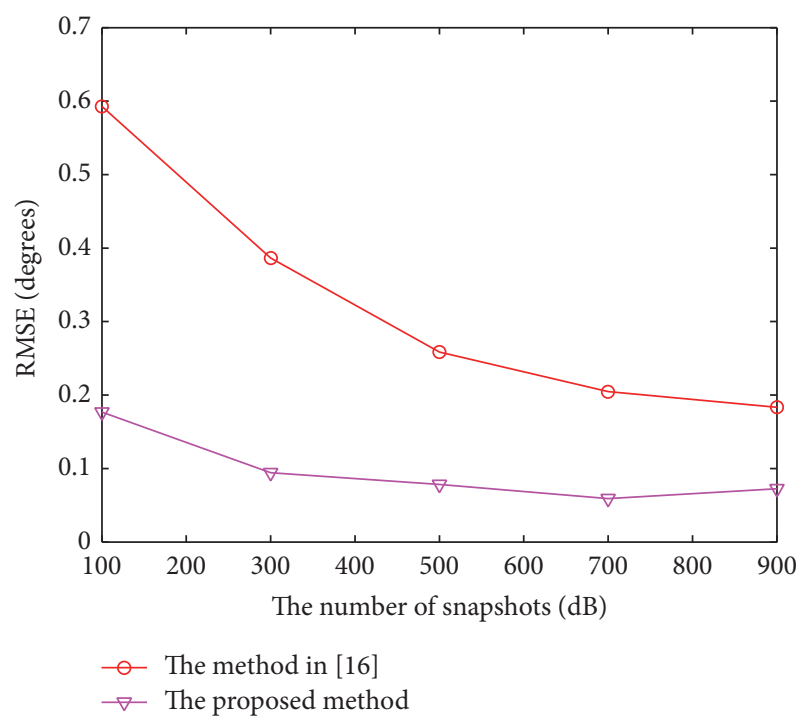

FIGURE 6: RMSE of DOA estimation with respect to the number of snapshots.

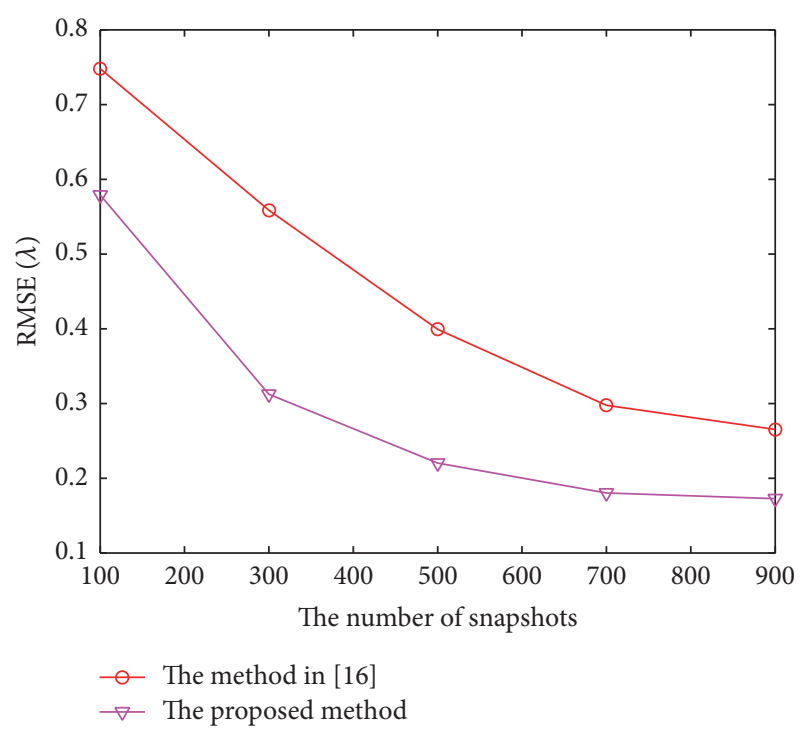

FIGURE 7: RMSE of range estimation with respect to the number of snapshots.

two figures, it can be discovered that the proposed method shows a lower RMSE than the method in [16] for different number of snapshots.

6.4. Resolution Ability versus SNR. In this subsection, the angular resolution ability regarding SNR is investigated. Two sources are defined to be resolved in a run if both $\left|\widehat{\theta}_{1}-\theta_{1}\right|$ and $\left|\widehat{\theta}_{2}-\theta_{2}\right|$ are smaller than $\left|\theta_{1}-\theta_{2}\right| / 2$, where $\widehat{\theta}_{i}$ and $\theta_{i}$ denote the estimate DOA and the real DOA, respectively. Consider a case in which two closely spaced signals located in $\left\{17 \lambda,-1.5^{\circ}\right\}$ and $\left\{11 \lambda, 1.5^{\circ}\right\}$ are imposed on a ULA with 15 elements and intersensor spacing $\lambda / 4$. By varying SNR from $0 \mathrm{~dB}$ to $18 \mathrm{~dB}$ with a step $2 \mathrm{~dB}$, the angular resolution ability for the above two methods is shown in Figure 8. It can

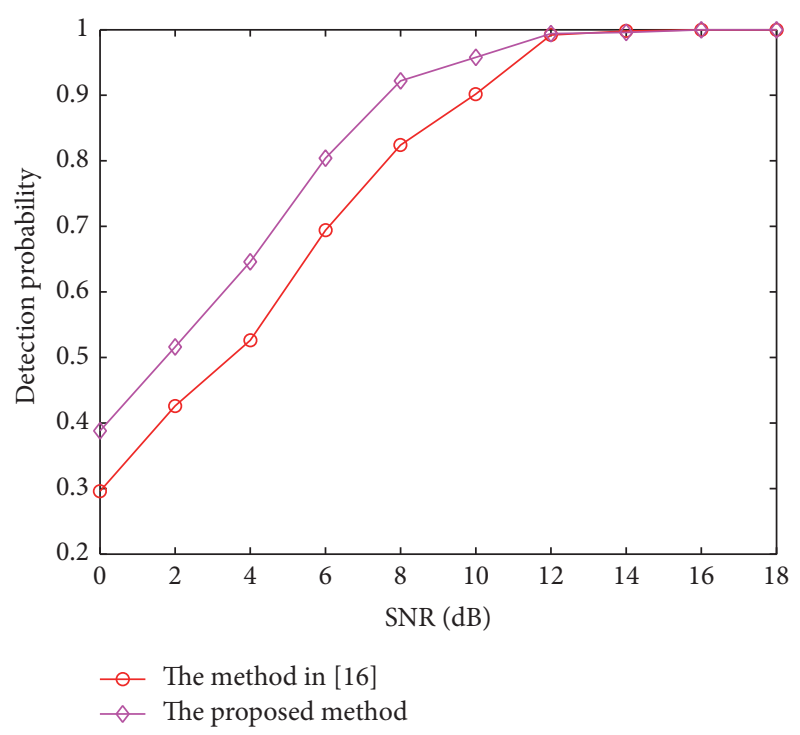

FIGURE 8: Angular resolution ability as a function of SNR.

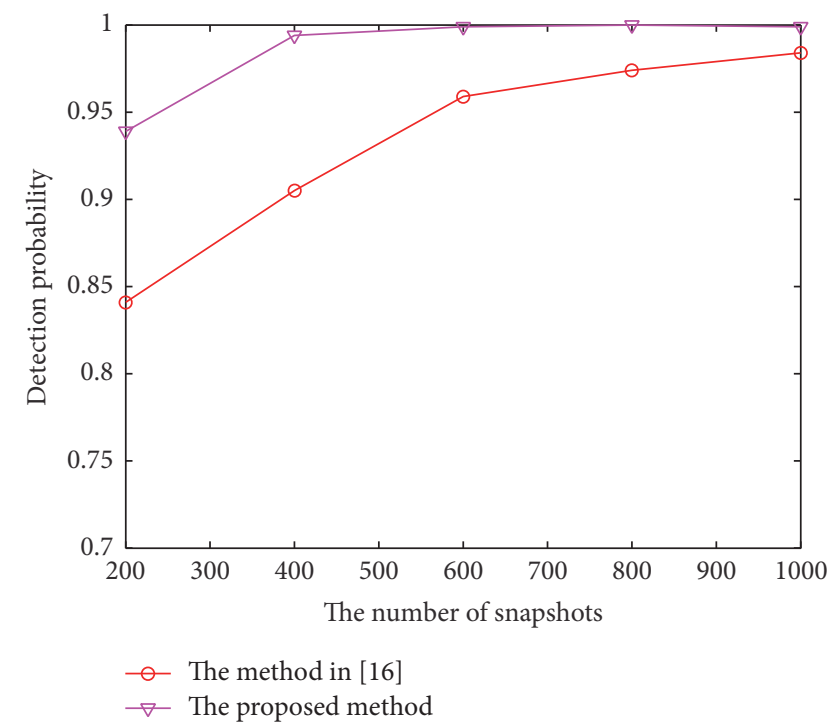

FIgURE 9: Angular resolution ability as a function of the number of snapshots.

be clearly noted that the proposed method achieves higher resolution than the method in [16].

6.5. Resolution Ability versus the Number of Snapshots. Now, we assess the angular resolution ability for the above two methods as a function of the number of snapshots. The parameters used in this experiment are kept the same as the previous one except $\mathrm{SNR}=10 \mathrm{~dB}$. Figure 9 shows the resolution ability versus the number of snapshots. The results in Figure 9 indicates that the proposed method has higher resolution compared to the method in [16].

According to the results from the above simulation experiments, it can be concluded that the proposed method shows a better performance compared to the method in [16] 
in terms of both RMSE and resolution ability, mainly because the idea of $\ell_{1}$-regularized weighted least-squares is utilized in the proposed method.

\section{Conclusions}

In this paper, a novel near-field source localization approach is proposed for a uniform linear array. Firstly, just like the method in [16], we convert a two-dimensional source localization problem into a one-dimensional DOA estimation one by employing the correlations of symmetric sensors of the array. Then, the method of $\ell_{1}$-regularized weighted leastsquares is exploited to estimate the DOAs of the sources. Meanwhile, a theoretical guidance for selecting the regularization parameter is also presented. At length, the L1-SVD method is used to find the ranges of sources based on the estimated DOAs. Future research includes low computational complexity method for source localization based on sparse signal recovery since the computational cost of the proposed method is a little high.

\section{Conflicts of Interest}

The authors declare that there are no conflicts of interest regarding the publication of this paper.

\section{Acknowledgments}

This work was supported in part by Chongqing Research Program of Basic Research and Frontier Technology of China under Grant nos. cstc2015jcyjA40055 and cstc2016jcyjA0515 and in part by Chongqing Municipal Education Commission of China under Grant nos. KJ1500917 and KJ1600936.

\section{References}

[1] J. C. Chen, R. E. Hudson, and K. Yao, "Maximum-likelihood source localization and unknown sensor location estimation for wideband signals in the near-field," IEEE Transactions on Signal Processing, vol. 50, no. 8, pp. 1843-1854, 2002.

[2] Y. D. Huang and M. Barkat, "Near-field multiple source localization by passive sensor array," IEEE Transactions on Antennas and Propagation, vol. 39, no. 7, pp. 968-975, 1991.

[3] R. Challa and S. Shamsunder, "High-order subspace-based algorithms for passive localization of near-field sources," in Proceedings of the 1995 Conference Record of the Twenty-Ninth Asilomar Conference on Signals, Systems and Computers, pp. 777-781, Pacific Grove, Calif, USA.

[4] N. Yuen and B. Friedlander, "Performance analysis of higher order ESPRIT for localization of near-field sources," IEEE Transactions on Signal Processing, vol. 46, no. 3, pp. 709-719, 1998.

[5] J. Liang and D. Liu, "Passive localization of mixed near-field and far-field sources using two-stage MUSIC algorithm," IEEE Transactions on Signal Processing, vol. 58, no. 1, pp. 108-120, 2010.

[6] J. Liang and D. Liu, "Passive localization of near-field sources using cumulant," IEEE Sensors Journal, vol. 9, no. 8, pp. 953960, 2009.
[7] S. Li and D. Xie, "Compressed symmetric nested arrays and their application for direction-of-arrival estimation of nearfield sources," Sensors, vol. 16, article 1939, no. 11, 2016 pages.

[8] E. Grosicki, K. Abed-Meraim, and Y. Hua, "A weighted linear prediction method for near-field source localization," IEEE Transactions on Signal Processing, vol. 53, no. 10, part 1, pp. 36513660, 2005.

[9] K. Abed-Meraim, Y. Hua, and A. Belouchrani, "Second-order near-field source localization: algorithm and performance analysis," in Procceding of the Conference Record of the 30th Asilomar Conference on Signals, Systems and Computers, pp. 723-727, 1996.

[10] W. J. Zhi and M. Y. Chia, "Near-field source localization via symmetric subarrays," in Proceedings of the IEEE International Conference on Acoustics, Speech and Signal Processing (ICASSP '07), vol. 2, pp. II-1121-II-1124, Honolulu, Hawaii, USA, April 2007.

[11] J. Xie, H. Tao, X. Rao, and J. Su, "Comments on 'near-field source localization via symmetric subarrays," IEEE Signal Processing Letters, vol. 22, no. 5, pp. 643-644, 2015.

[12] D. Malioutov, M. Çetin, and A. S. Willsky, "A sparse signal reconstruction perspective for source localization with sensor arrays," IEEE Transactions on Signal Processing, vol. 53, no. 8, part 2, pp. 3010-3022, 2005.

[13] B. Wang, J. Liu, and X. Sun, "Mixed sources localization based on sparse signal reconstruction," IEEE Signal Processing letters, vol. 19, no. 8, pp. 487-490, 2012.

[14] S. Qin, Y. D. Zhang, Q. Wu, and M. G. Amin, "Structure-aware bayesian compressive sensing for near-field source localization based on sensor-angle distributions," International Journal of Antennas and Propagation, vol. 2015, Article ID 783467, 15 pages, 2015.

[15] Y. Tian and X. Sun, "Passive localization of mixed sources jointly using MUSIC and sparse signal reconstruction," AEU-International Journal of Electronics and Communications, vol. 68, no. 6, pp. 534-539, 2014.

[16] K. Hu, S. P. Chepuri, and G. Leus, "Near-field source localization using sparse recovery techniques," in Proceedings of the International Conference on Signal Processing and Communications (SPCOM '14), pp. 1-5, Bangalore, India, July 2014.

[17] K. Hu, S. P. Chepuri, and G. Leus, "Near-field source localization: sparse recovery techniques and grid matching," in Proceedings of the IEEE 8th Sensor Array and Multichannel Signal Processing Workshop (SAM '14), pp. 369-372, A Coruña, Spain, June 2014.

[18] J. Yin and T. Chen, "Direction-of-arrival estimation using a sparse representation of array covariance vectors," IEEE Transactions on Signal Processing, vol. 59, no. 9, pp. 4489-4493, 2011.

[19] B. Ottersten, P. Stoica, and R. Roy, "Covariance matching estimation techniques for array signal processing applications," Digital Signal Processing, vol. 8, no. 3, pp. 185-210, 1998.

[20] M. Grant, S. Boyd, and Y. Ye, "CVX: Matlab software for disciplined convex programming," http://cvxr.com/cvx/. 


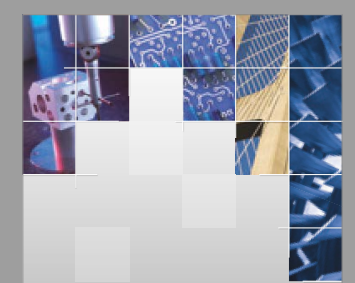

\section{Enfincering}
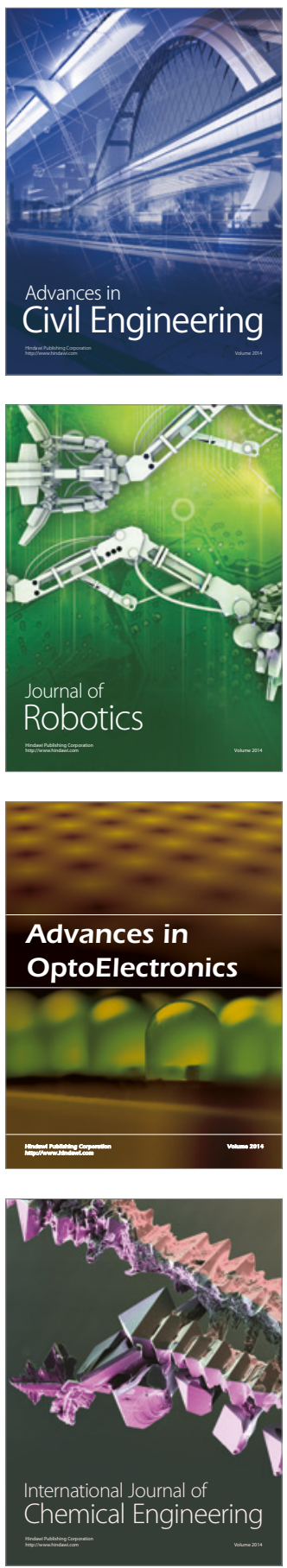

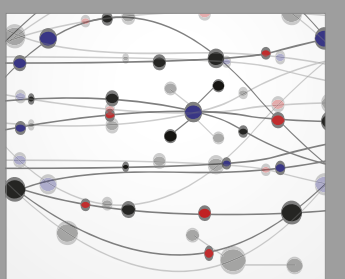

The Scientific World Journal

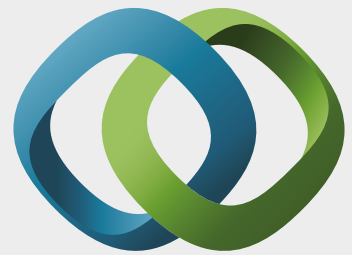

\section{Hindawi}

Submit your manuscripts at

https://www.hindawi.com
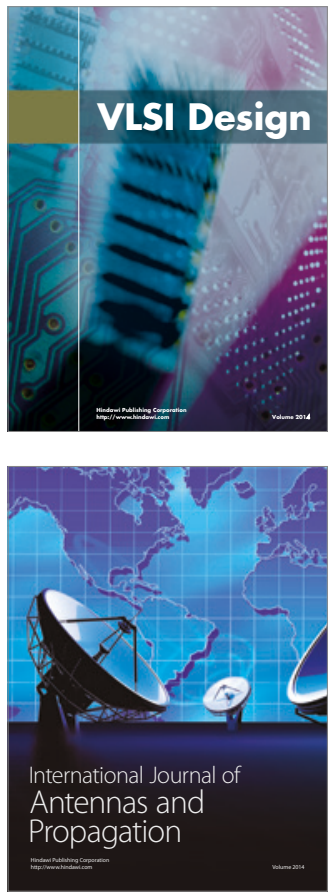

\section{Rotating}

Machinery
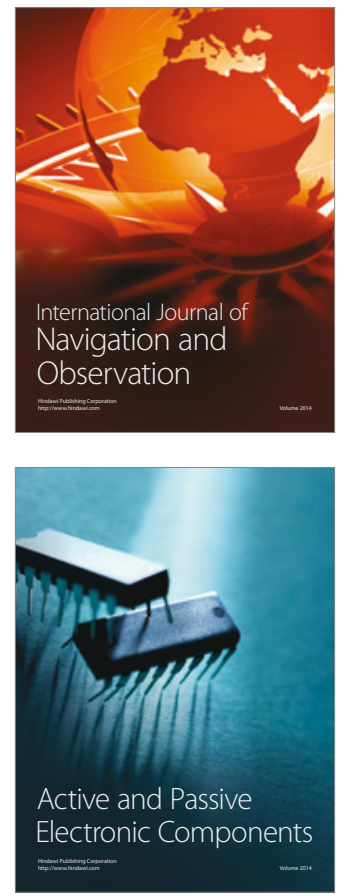
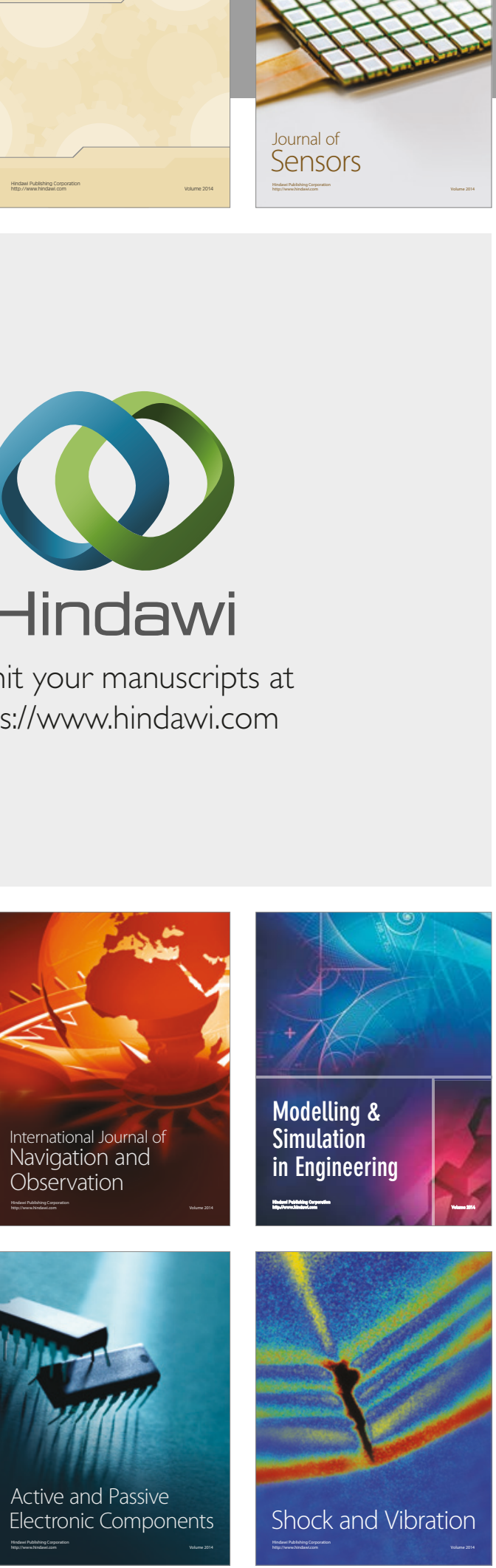
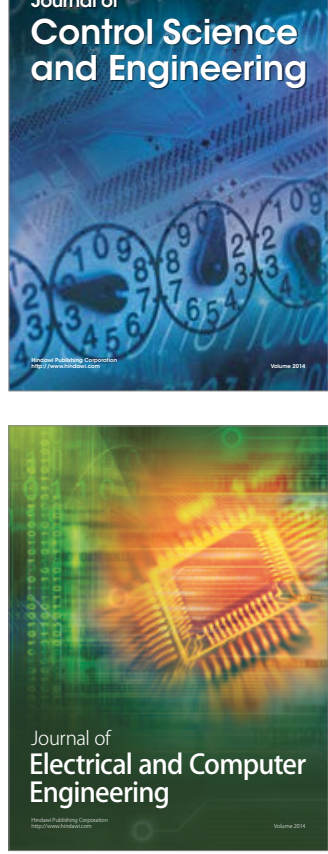

Distributed

Journal of

Control Science

and Engineering
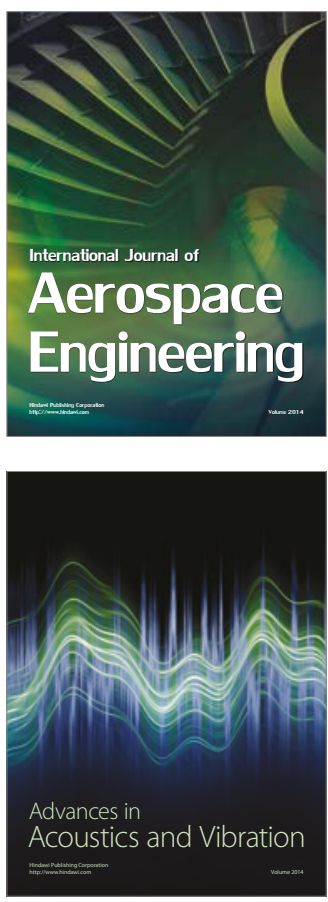

Sensor Networks 